\title{
A NEW CHARACTERIZATION OF HEREDITARY ALGEBRAS
}

\author{
YICHAO YANG AND JINDE XU
}

\begin{abstract}
In this short paper we prove that a finite dimensional algebra is hereditary if and only if there is no loop in its ordinary quiver and every $\tau$-tilting module is tilting.
\end{abstract}

\section{INTRODUCTION}

Throughout this paper, $A$ stands for a finite dimensional basic algebra over an algebraically closed field $k$ and $Q_{A}$ for its ordinary quiver. The $\tau$-tilting theory was recently introduced by Adachi, Iyama and Reiten in [1], which completes the classical tilting theory from the viewpoint of mutation. Note that a tilting $A$-module is always $\tau$-tilting and the converse is true if $A$ is hereditary.

It is therefore interesting to consider whether the heredity of $A$ can be characterized by the property that every $\tau$-tilting $A$-module is tilting. Indeed, it is not true as shown by the following example. Let $A$ be a non-simple local finite dimensional $k$-algebra, then $A$ is the only $\tau$-tilting $A$-module which is also tilting but $A$ is not hereditary. In this counterexample we observe that there exists a loop in $Q_{A}$, thus it is necessary to add some extra restrictions to our assumptions.

Our main result says that a finite dimensional algebra $A$ is hereditary if and only if there is no loop in $Q_{A}$ and every $\tau$-tilting $A$-module is tilting.

\section{Main Result}

In what follows, let mod- $A$ be the category of finitely generated right $A$-modules. For $M \in$ mod$A$, we denote by add $M$ (respectively, Fac $M$ ) the category of all direct summands (respectively, factor modules) of finite direct sums of copies of $M$. Moreover, we denote by $|M|$ the number of pairwise non-isomorphic indecomposable direct summands of $M$. Also, we denote by $P_{i}$ (respectively, $S_{i}$ ) the indecomposable projective (respectively, simple) $A$-module associated to $i$ for each vertex $i \in\left(Q_{A}\right)_{0}$. Unless stated otherwise, all modules are assumed to be basic right modules.

Recall that an $A$-module $T$ is tilting if it satisfies:

(1) $\operatorname{pd}_{A} T \leq 1$,

(2) $\operatorname{Ext}_{A}^{1}(T, T)=0$ and,

(3) there is an exact sequence $0 \rightarrow A \rightarrow T_{0} \rightarrow T_{1} \rightarrow 0$ with $T_{0}, T_{1} \in$ add $T$.

It is well known that the condition (3) can be replaced by (3') $|T|=|A|$.

From [1] we also recall the following definitions in $\tau$-tilting theory.

(1) $M$ in mod- $A$ is called $\tau$-rigid if $\operatorname{Hom}_{A}(M, \tau M)=0$, where $\tau$ is the Auslander-Reiten translation functor.

(2) $M$ in mod- $A$ is called $\tau$-tilting if $M$ is $\tau$-rigid and $|M|=|A|$.

2010 Mathematics Subject Classification: 16G20, 16E10.

Keywords: Tilting module, $\tau$-tilting module, Hereditary algebras. 
(3) $M$ in mod- $A$ is called support $\tau$-tilting if there exists an idempotent $e$ of $A$ such that $M$ is a $\tau$-tilting $(A /\langle e\rangle)$-module.

The main result of this paper is the following.

Theorem 2.1. Let $A$ be a finite dimensional algebra. Then $A$ is hereditary if and only if there is no loop in $Q_{A}$ and every $\tau$-tilting A-module is tilting.

Proof. First we assume that $A$ is a hereditary algebra. Then for any $A$-module $M$, its projective dimension $\operatorname{pd}_{A} M \leq 1$. Thus every $\tau$-tilting $A$-module is tilting. Note that $A$ is also a finite dimensional algebra, it is clear that there is no loop in $Q_{A}$.

On the other hand if $A$ is not a hereditary algebra, then its global dimension $\operatorname{gl} \cdot \operatorname{dim} . A>1$. Because the global dimension of $A$ is also equals to the supremum of the set of projective dimensions of all simple $A$-modules, there exists a simple $A$-module $S_{i}$ such that $\operatorname{pd}_{A} S_{i}>1$. For simplicity, we may take $i=1$.

Since there is no loop in $Q_{A}$, we have $\operatorname{Ext}_{A}^{1}\left(S_{1}, S_{1}\right)=0$. Moreover, it can easily be seen that Fac $S_{1}=$ add $S_{1}$, hence $\operatorname{Ext}_{A}^{1}\left(S_{1}\right.$, Fac $\left.S_{1}\right)=0$. Now by [3], Proposition 5.8] we have $\operatorname{Hom}_{A}\left(S_{1}, \tau S_{1}\right)=$ 0 , therefore the simple module $S_{1}$ is a $\tau$-rigid module.

According to [1], Theorem 2.10], it follows that $S_{1}$ can be completed to a $\tau$-tilting module, that is, there exists another $A$-module $U$ such that $S_{1} \oplus U$ is a $\tau$-tilting module. On the other hand, we have $\operatorname{pd}_{A}\left(S_{1} \oplus U\right)=\max \left\{\operatorname{pd}_{A} S_{1}, \operatorname{pd}_{A} U\right\} \geq \operatorname{pd}_{A} S_{1}>1$, hence $S_{1} \oplus U$ is not a tilting module. Finally we find a $\tau$-tilting $A$-module $S_{1} \oplus U$ but not tilting, which contradicts our assumption. The proof of the theorem is now complete.

From now on we will give some applications of Theorem 2.1. Firstly, the famous no loop conjecture stated in [5] affirms that the ordinary quiver $Q_{A}$ of $A$ contains no loop if $A$ is of finite global dimension, while the strong no loop conjecture, strengthens this to state that a vertex in the ordinary quiver $Q_{A}$ admits no loop if it has finite projective dimension, see [2, 5]. Since for a finite dimensional algebra $A$ both these two conjectures have been proved in [6], immediately we have the following application.

Corollary 2.2. Let A be a finite dimensional algebra of finite global dimension. Then A is hereditary if and only if every $\tau$-tilting A-module is tilting.

Recall from [4] that an $A$-module $M$ is faithful if its right annihilator Ann $M=\{a \in A \mid M a=0\}$ vanishes and $M$ is sincere if $\operatorname{Hom}_{A}(P, M) \neq 0$ for any projective $A$-module $P$. It is easy to see that any faithful module is sincere.

According to [[1, Proposition 2.2] it follows that $\tau$-tilting modules are precisely sincere support $\tau$-tilting modules and tilting modules are precisely faithful support $\tau$-tilting modules, hence to some extent the difference between $\tau$-tilting modules and tilting modules is analog of the difference between faithful modules and sincere modules. Now we have the following direct consequence, which is another application of Theorem 2.1.

Corollary 2.3. Let $A$ be a finite dimensional algebra with no loop in $Q_{A}$. If every sincere $A$ module is faithful, then A is hereditary.

However, the converse of Corollary 2.3 is not true in general. Let $A$ be the Kronecker algebra and $\mathcal{T}^{A}=\left\{\mathcal{T}_{\lambda}^{A}\right\}_{\lambda \in \mathbb{P}_{1}(k)}$ be the $\mathbb{P}_{1}(k)$-family of pairwise orthogonal standard stable tubes, then $A$ is a hereditary algebra and all modules lying on the mouth of the rank one tubes of $\mathcal{T}^{A}$ are sincere $A$-modules but not faithful, see [8]. 
Indeed, in [7, Corollary 2.3] Ringel have shown the following conclusion. Let $A$ be a hereditary algebra and $M$ be a sincere $A$-module with no self-extensions, then $M$ is also a faithful module. Conversely we have the following result.

Corollary 2.4. Let $A$ be a finite dimensional algebra with no loop in $Q_{A}$. If every sincere $A$ module with no self-extensions is faithful, then A is hereditary.

Proof. By Theorem 2.1 it suffices to show that every $\tau$-tilting $A$-module is tilting. Let $M$ be any $\tau$-tilting $A$-module, then by [1], Proposition 2.2] $M$ is a sincere support $\tau$-tilting module. Thus $M$ is a $\tau$-rigid module, $\operatorname{Hom}_{A}(M, \tau M)=0$ and hence $\operatorname{Ext}_{A}^{1}(M, M)=0$, i.e., $M$ has no self-extensions. Now $M$ is a sincere $A$-module with no self-extensions, which is faithful by our assumption. Consequently $M$ is a faithful support $\tau$-tilting module. According to [1], Proposition 2.2] again it follows that $M$ is actually a tilting module, which completes the proof.

Using this, the following is now a direct consequence.

Proposition 2.5. Let A be a finite dimensional algebra. Then $A$ is hereditary if and only if there is no loop in $Q_{A}$ and every sincere A-module with no self-extensions is faithful.

We end with the following easy example.

Let $A$ be the algebra given by quiver $1 \stackrel{\alpha}{\longrightarrow} 2 \stackrel{\beta}{\longrightarrow} 3$ with the relation $\alpha \beta=0$. Then $A$ is not a hereditary algebra and there is no loop in $Q_{A}$. Thus by Theorem 2.1 there exists some $\tau$-tilting $A$-modules which are not tilting.

Indeed, it is easy to see that the class of tilting $A$-modules is $\left\{P_{1} \oplus P_{2} \oplus P_{3}, P_{1} \oplus P_{2} \oplus S_{2}\right\}$, while the class of $\tau$-tilting $A$-modules is $\left\{P_{1} \oplus P_{2} \oplus P_{3}, P_{1} \oplus P_{2} \oplus S_{2}\right\} \cup\left\{P_{1} \oplus P_{3} \oplus S_{1}\right\}$ since $\operatorname{pd}_{A} S_{1}=2>1$.

Acknowledgements. The first author is supported by Fonds Québécois de la Recherche sur la Nature et les Technologies (Québec, Canada) through the Merit Scholarship Program For Foreign Students. This work was carried out when both authors are postdoctoral fellows at Université de Sherbrooke. They would like to thank Prof. Shiping Liu for his helpful discussions and warm hospitality.

\section{REFERENCES}

[1] T. Adachi, O. Iyama and I. Reiten, $\tau$-tilting theory, Compos. Math. 150 (2014), no. 3, 415-452.

[2] M. Auslander, I. Reiten and S. O. Smalø, Representation theory of Artin algebras, Cambridge Studies in Advanced Mathematics, 36. Cambridge University Press, Cambridge, 1995.

[3] M. Auslander and S. O. Smalø, Almost split sequences in subcategories, J. Algebra 69 (1981), no. 2, 426-454. Addendum; J. Algebra 71 (1981), no. 2, 592-594.

[4] I. Assem, D. Simson and A. Skowroński, Elements of the representation theory of associative algebras. Vol. 1. Techniques of representation theory, London Mathematical Society Student Texts, 65. Cambridge University Press, Cambridge, 2006.

[5] K. Igusa, Notes on the no loops conjecture, J. Pure Appl. Algebra 69 (1990), no. 2, 161-176.

[6] K. Igusa, S. Liu and C. Paquette, A proof of the strong no loop conjecture, Adv. Math. 228 (2011), no. 5, 2731-2742.

[7] C. M. Ringel, Exceptional objects in hereditary categories, Representation theory of groups, algebras and orders (Constanta, 1995), An. Stiint. Univ. Ovidius Constanta Ser. Mat. 4 (1996), no. 2, 150-158.

[8] D. Simson and A. Skowroński, Elements of the representation theory of associative algebras. Vol. 2. Tubes and concealed algebras of Euclidean type, London Mathematical Society Student Texts, 71. Cambridge University Press, Cambridge, 2007. 
Département de mathématiques, Université de Sherbrooke, Sherbrooke, Québec, Canada, J1K 2R1

E-mail address: yichao.yang@usherbrooke.ca

Département de mathématiques, Université de Sherbrooke, Sherbrooke, Québec, Canada, J1K 2R1

E-mail address: jinde.xu@usherbrooke.ca 